\title{
Index Vol. 17, 1986
}

\begin{tabular}{c|c|c|}
\begin{tabular}{c|c} 
No. \\
Month
\end{tabular} & 1 & 2 \\
Issue & January & February \\
Biophysics & Physics \\
Pages & $1-16$ & $17-32$
\end{tabular}

Applied Physics, Physics in Industry

Advisory Committee, 79

Electroluminescence, thin film displays, 17-20

Femtosecond laser pulses, generation and applications, 105-108

Semiconductor Superlattices and quantum well structures, artificial, 44-47

Workshop on low resolution, pyroelectric arrays, 98

Astronomy and Astrophysics

Divisional and Section Boards, 80, 98

International research facilities, seminar, 61-62

$\mathrm{X}$-ray binaries, 21-24

Atomic and Molecular Physics

Charge exchange in collisions; experiment, 66-69 theory, 83-87

Divisional and Section Boards, 80

Photophysical processes, elementary molecular beam studies, $89-93$

Biophysics,

Electrostatic forces, long range, 8-10

Microcalorimetry and thermodynamics, $14-16$

Muscle contraction, 5-7

NMR with proteins and nucleic acids, 11-13

Physicists, role in biology, 1-2

Protein structure, 3-4

Condensed Matter Physics

Blue phases, frustrated liquids, 113-115

Charge density wave, sliding, induces electron

transport, 99-103

Crystal surfaces, roughness of, 136-140

Divisional Boards and Section Committees, 80

6th General Conference, 97-98

International research facilities seminar, 62

Macroscopic quantum phenomena in Josephson elements, 94-96

Magnetism of ferromagnetic materials above Curie temperature, $25-28$

Neutron spin echo spectroscopy, applications in magnetism, 129-131

Scanning Tunnelling Microscopy,

Nobel Prizes 1986, 141-144

Semiconductor superlattices and quantum well structures, artificial, 44-47

Who's Who, 121

S. Balibar, $136-140$

A. Baratoff, 141-144

H.J.C. Berendsen, 8-10

C.-I. Bränden, 3-4

P.J. Brown, 25-28

W.R.G. Buckel, 57

P.E. Cladis, 113-115
3

Meetings

$33-40$

4
April
Physics
$41-56$

\begin{tabular}{c|c|}
5 & 6 \\
May & June \\
Physics & Physics \\
& + Directory \\
$57-72$ & $73-88$
\end{tabular}

\section{Principal Subjects}

Conferences

Advisory Committee, 79

7 th EPS General, 121

International research facilities, 60-62 proceedings, 121

Laser-plasma phenomena, 70-71

Meeting and schools, 33-40, 121-128

Workshop for Associate Members, 98

Education, Advisory Committee, 79

European Physical Society

Associate Members, 8

Conferences

7 th General, 121

International research facilities, 60-62, 121

Constitution changes, 56

Council report, 58-59

Delegates, IOM, 56

Associate Members, 77

Directory, 77-82

Executive Committee, 56

Lecturer, 1986 tour, 32

Members, 47, 77-78, 98

President, message from, 57

Staff, 120

Fractals in physics, 41-43

Hewlett-Packard Europhysics Prize

Announcement of 1986 award, 16

Call for nominations, 88

Neutron spin echo spectroscopy, applications in magnetism,

High Energy and Particle Physics

Divisional Board, 81

International research facilities, seminar, 62

Proceedings of Bari conferences, 32

Hydrogen plasma-phase diagram and properties, $52-55$

Inter-Divisional Groups

Computational Physics, Board, 81

Physics for Development, development for physics, 30-31

Board, 81

Experimental Physics Control Systems, 72

Board, 81

\section{Authors}

H. Hora, 70-71

H.E. Huxley, 5-7

S.C. Jardin, 73-76

E. Kellenberger, 1-2

W.D. Kraeft, 52-55

D. Kremp, 52-55

P. Laporta, 105-108
E. Lillethun, 30-31

C. de Loore, 21-24

R. Loudon, 109-112

R. Mach, 17-20

A.R. Mackintosh, 97-98

V. Magni, $105-108$

F. Mezei, 129-131

\begin{tabular}{c|c|c|c|}
$7 / 8$ & 9 & 10 & $11 / 12$ \\
July/Aug. & September & October & Nov./Dec. \\
Physics & Physics & Meetings & Physics \\
$89-104$ & $105-120$ & $121-128$ & $129-144$
\end{tabular}

ICTP, donation programme, 51

International Radiation Physics Society, 104

International workshops, Hungarian centre, 33

Japan, Physical Society of, 104

Magnetism

Ferromagnetic materials above Curie

temperature, $25-28$

Megagauss fields available for research, 29

Neutron spin echo spectroscopy, 129-131.

Nobel Prizes 1986, 141-144

Nuclear Physics

Divisional Board, 81

International research facilities, seminar, 60-61

Optics

Divisional Board, 8

Non-classical light, 109-112

Physics and Society

Advisory Committee, 79

Inter-Divisional Group for Development, 30-31, 81

Plasma Physics (see also Thermonuclear Fusion)

Divisional Board, 81, 98

Hydrogen phase diagram and properties, 52-55

Non-linear waves in, 63-65

Publications

Advisory Committee, 79

EPS prices, 120

Europhysics Conference Abstracts, 33

Europhysics Letters, subscriptions, 32, 104

HEPP, Proceedings of Bari, 32

Quantum Electronics

Divisional Board, 8

Laser-generated intense Planck radiation, 116-120

Laser pulses, fs, generation and

applications, 105-108

Non-classical light, 109-112

Thermonuclear Fusion (see also Plasma Physics)

Asdex, $\mathrm{H}$-mode of, 48-51

JET Experiment, Progress with, 132-125

Laser fusion, boost for, 70-71

Laser-generated intense Planck radiation, 117-120

Spheromak, 73-76

G.H. Miley, 70-71

P. Monceau, 99-103

G.O. Müller, 17-20

P. Pieranski, 113-115

K. Ploog, 44-47

M. Renard, 99-103

G. Schön, 94-96
P.E. Stott, 132-135

V. Sidis, 66-69, 83-87

R. Sigel, 117-120

S. De Silvestri, 105-108

K.H. Spatschek, 63-65

F. Wagner, 48-51

K. Wüthrich, 11-13
EPS Divisions, Sections and Group

Astronomy and Astrophysics Division

Solar Physics Section

Atomic and Molecular Physics Division

Atomic Spectroscopy Section

Chemical Physics

Electronic and Atomic Collisions

Molecular Physics

Computational Physics Group

Condensed Matter Division

Liquids Section

Low Temperature Physics Section

Macromolecular Physics

Macromolec
Magnetism

Metal Physics

Metal Physics

Surfaces and Interfaces

High Energy \& Particle Physics Division

Interdiv, Group on Exptl. Phys. Control Systems

Interdiv. Group on Physics for Development

Nuclear Physics Division

optics Division

Plasma Physics Division

Quantum Electronics Division
Europhysics News is the official journal of the European Physical Society which comprises 29 National Societies, Academies and Group, about 4000 Individual Members and 70 Associate Members. Governing bo dies of EPS are the General Meeting, Council and an elected Executive Committee responsible for detailed policy. EPS promotes the collaboration of physicists policy. EPS promotes the collaboration of physicists rences and publications, improving physics education encouraging physics applications, awarding scholarships to sponsored schools in Erice. EPS publishes in addition to Europhys. News, Europhysics Letters lin partnership with national societies), European Journal partnership with national societies), European Joumal of Physics (in partnership with The UK Inst. of Phys.), Members receive Europhys. News free of charge (price to insts.: Sw.Fr. 90.-/a), Europhys. Lett. at Sw.Fr. 70.-1a (insts. 590.-), rebates on many other publications and on conference fees. Annual EPS membership fee for In. on conference fees. Annual EPS membership fee for Individual Members belonging to an EPS member society members of a Collaborating Society: Sw.Fr. 55 .

\section{Editor: E.N. Shaw}

Editorial Board:

A. Baratoff, F. James, M. Lohmann,

M. Mayor, J. Muller, M. Siegrist

Editorial and Advertising Office at the EPS Secretariat

Address: EUROPEAN PHYSICAL SOCIETY P.O. Box 69 , CH- 1213 Petit-Lancy 2 Switzerland

Telephone: Geneva (22) 931130

Telex: 428024 eps ch

Cables: europhys genève

Printed by: Pfirter frères sa CH-1213 Petit-Lancy/Switzerland 